\title{
Consumo de Vitaminas Antioxidantes por Mulheres com Câncer de Mama submetidas ao Tratamento Quimioterápico na Cidade de Pelotas-RS
}

\author{
Consumption of Antioxidant Vitamins by Women with Breast Cancer Undergoing \\ Chemotherapy Treatment in the City of Pelotas-RS \\ Consumo de Vitaminas Antioxidantes por parte de Mujeres con Cáncer de Mama \\ sometidas al Tratamiento de Quimioterapia en la Ciudad de Pelotas-RS
}

Tassia Ney Portantiolo하 ; Idrejane Aparecida Viccari do Vale ${ }^{2}$; Rafaela Bülow Bergmann ${ }^{3}$; Renata Torres Abib ${ }^{4}$

\section{Resumo}

Introdução: $\mathrm{O}$ câncer de mama é o mais comum no sexo feminino e, no Sul, tem uma das taxas mais altas dos do país: afeta mais de 70 a cada 100.000 mulheres. Objetivo: Avaliar o consumo de vitaminas antioxidantes por mulheres com câncer de mama em tratamento quimioterápico e verificar a necessidade de adequar o seu consumo. Método: Estudo transversal com pacientes com neoplasia mamária em quimioterapia no Setor de Oncologia do Hospital Escola da Universidade Federal de Pelotas, atendidas no período de maio a dezembro de 2012. O consumo alimentar das pacientes foi avaliado por Questionário de Frequência Alimentar. Resultados: A amostra foi formada por 23 mulheres, 78,3\%

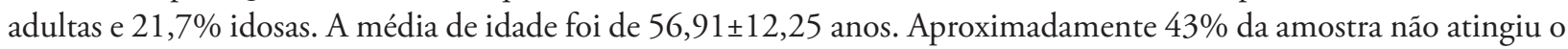
valor recomendado de ingestão de Vitamina A, embora a mediana de consumo tenha sido maior que a recomendação. Em relação à Vitamina C, todas ultrapassaram o ideal, enquanto nenhuma consumiu quantidade mínima de vitamina E. Conclusão: Observou-se que existe um consumo diminuído de vitamina E por todas as pacientes, e um número expressivo de mulheres com baixo consumo de vitamina A, importantes antioxidantes da dieta que podem contribuir para neutralizar o perfil pró-oxidativo da doença. Notou-se a necessidade de aconselhamento nutricional, a fim de adequar o consumo de vitaminas antioxidantes na dieta, com o intuito de auxiliar no tratamento e melhorar o estado nutricional dessas pacientes.

Palavras-chave: Neoplasias da Mama; Vitaminas; Quimioterapia; Humanos; Feminino; Brasil

\footnotetext{
${ }^{1}$ Nutricionista pela Universidade Federal de Pelotas (UFPel). Residente do Programa de Residência Integrada Multiprofissional em Atenção à Saúde Oncológica da UFPel. Pelotas (RS), Brasil. E-mail: tassianp@hotmail.com.

${ }^{2}$ Nutricionista pela UFPel. Mestranda do Programa de Pós-graduação em Nutrição e Alimentos pela UFPel. Pelotas (RS), Brasil. E-mail: idrejanev@yahoo.com.br.

${ }^{3}$ Nutricionista pela UFPel. Mestranda do Programa de Pós-graduação em Saúde e Comportamento pela UFPel. Pelotas (RS), Brasil. E-mail: bergmann.rafa@gmail.com.

${ }^{4}$ Nutricionista pela Pontifícia Universidade Católica do Rio Grande do Sul (PUCRS). Doutora em Ciências Biológicas pela Universidade Federal do Rio Grande do Sul (UFRGS). Professora-Associada da Faculdade de Nutrição da UFPel. Pelotas (RS), Brasil. E-mail: renata.abib@ymail.com.

Enderę̧o para correspondência: Tássia Ney Portantiolo. Rua Lindolfo Collor, 30 - Três Vendas. Pelotas (RS), Brasil. CEP: 96020-470. E-mail: tassianp@hotmail.com.
} 


\section{INTRODUÇÃO}

No Brasil, estimam-se 395 mil casos novos de câncer para o ano de 2015. O câncer de mama é o mais comum no sexo feminino e, no Sul, tem uma das taxas mais altas do país: afeta mais de 70 a cada 100.000 mulheres ${ }^{1}$.

Uma das formas mais comuns de tratamento para esse tipo de câncer é a quimioterapia. Considerada um tratamento sistêmico ${ }^{2}$, consiste em destruir as células malignas, preservando as normais; porém, alguns agentes quimioterápicos atuam de forma inespecífica e acabam atingindo as células sadias, gerando toxicidade em diversos tecidos ${ }^{3}$. Esse tipo de efeito oxidativo sobre as células sadias pode explicar, em parte, a presença dos efeitos colaterais comumente observados nos pacientes.

$\mathrm{O}$ estresse oxidativo está relacionado não somente à toxicidade do tratamento, mas também à própria etiologia do câncer, quando o excesso de espécies reativas de oxigênio (ERO) entra em desequilíbrio com a capacidade de defesa antioxidante do organismo ${ }^{4}$. No câncer, as ERO também podem estimular a proliferação celular por meio de mutaçóes sobre o DNA, causando progressão tumoral ${ }^{4}$. Entretanto, é possível prevenir esse desbalanço entre as moléculas antioxidantes e pró-oxidantes por meio da oferta de nutrientes como as vitaminas A, C e E, capazes de neutralizar as ERO. Desta forma, os antioxidantes podem auxiliar tanto na prevençáo do câncer de mama quanto no seu tratamento, contribuindo com a redução dos efeitos colaterais relacionados à quimioterapia ${ }^{5}$.

Recentemente foi observado que mulheres com câncer de mama apresentam alteraçóes do consumo alimentar e aumento dos níveis de marcadores de estresse oxidativo ${ }^{6}$. Quando submetidas ao tratamento quimioterápico, podem apresentar diminuída capacidade antioxidante endógena e aumento da peroxidação lipídica, indicando assim que o tratamento ou até mesmo a própria doença pode estar gerando o aumento do estresse oxidativo, o que justifica mais uma vez o adequado consumo de antioxidantes por esse público 5 .

$\mathrm{A}$ análise do consumo das vitaminas $\mathrm{A}, \mathrm{C}$ e $\mathrm{E}$ em mulheres com câncer de mama se torna extremamente relevante em função do seu alto poder antioxidante, sendo também uma forma de elucidar a necessidade de adequar a ingestão conforme a recomendaçấo desses nutrientes. Considerando que até o momento existem poucos dados na literatura que demonstrem essa análise, principalmente na população brasileira, o objetivo do presente estudo foi avaliar o consumo de vitaminas antioxidantes por mulheres com câncer de mama submetidas ao tratamento quimioterápico e verificar a necessidade de adequar o seu consumo.

\section{MÉTODO}

Trata-se de um estudo transversal, em que foram entrevistadas pacientes maiores de 18 anos com diagnóstico de câncer de mama primário durante a primeira sessão de quimioterapia, no Setor de Oncologia do Hospital Escola da Universidade Federal de Pelotas (UFPel), atendidas pelo Sistema Único de Saúde (SUS), entre maio e dezembro de 2012. Os dados foram coletados após o esclarecimento sobre a pesquisa e a leitura e assinatura do Termo de Consentimento Livre e Esclarecido. Foram excluídas pacientes em uso de terapia nutricional enteral e presença de outra neoplasia associada. Este trabalho foi derivado de um projeto de pesquisa aprovado pelo Comitê de Ética em Pesquisa da UFPel, no 32/12.

A frequência do consumo de alimentos fontes de vitaminas A, C e E foi avaliada a partir do Questionário de Frequência Alimentar $(\mathrm{QFA})^{7}$ desenvolvido para estudar fatores dietéticos nas doenças crônicas não transmissíveis, que tem como principal característica avaliar a ingestão alimentar do último mês em apenas uma aplicação. Para estabelecer a quantidade ingerida, em gramas, de cada alimento referido, considerou-se: o número de vezes em que o alimento foi ingerido segundo cada paciente; a frequência (diária, semanal ou mensal); e o tamanho da porção (pequena, média ou grande). Com base na quantidade total consumida de cada alimento, realizou-se a análise do total de cada vitamina consumida no mês. A mediana de consumo de cada vitamina antioxidante foi obtida através do programa SPSS ${ }^{\bullet}$ versão 10.0. $\mathrm{O}$ consumo diário foi comparado às recomendaçóes da Dietary Reference Intakes (DRI) ${ }^{8}$ para a referida faixa etária.

As análises de composição dos alimentos foram realizadas com base na Tabela Brasileira de Composição de Alimentos (TACO) ${ }^{9}$, na Tabela de Composição Química de Alimentos da Universidade Federal de São Paulo (Unifesp) ${ }^{10}$, na Tabela do Departamento de Agricultura dos Estados Unidos da América (United States Department of Agriculture - USDA $)^{11}$ e na informaçáo nutricional contida em rótulos alimentares.

As variáveis foram descritas como mediana e percentis, média e desvio-padrão ou percentuais e frequências relativas, conforme sua natureza. Para a análise, foi utilizado o programa estatístico SPSS ${ }^{\circledR}$ versão 10.0.

\section{RESULTADOS}

Das 27 pacientes que se enquadravam nos critérios de inclusão, 23 foram analisadas, havendo quatro perdas em função do não comparecimento no dia da quimioterapia. A média de idade da amostra foi de 56,91 $\pm 12,25$ anos, sendo $56,5 \%$ pacientes adultas e $43,5 \%$ idosas (idade $\geq$ que 60 anos).

A mediana de consumo de vitamina A foi de 934,69 $\mu \mathrm{g} / \mathrm{dia}(409,24-1.542,22)$, resultado superior à recomendaçáo da $\mathrm{DRI}^{1}$, que preconiza um consumo diário de $700 \mu \mathrm{g} / \mathrm{dia}$. A vitamina $\mathrm{C}$ apresentou valores de consumo diário acima do recomendado, com mediana de $250,36 \mathrm{mg} / \mathrm{dia}(131,02-498,72)$. Para a vitamina E, 
a mediana de consumo diário foi de $3,27 \mathrm{mg} / \mathrm{dia}(2,32-$ $5,42)$, valor abaixo do recomendado ( $15 \mathrm{mg} / \mathrm{dia})$, como apresentado na Tabela 1.

Observou-se que $43,47 \%$ das pacientes náo apresentaram consumo diário adequado de vitamina $\mathrm{A} e$ nenhuma atingiu consumo diário adequado de vitamina $\mathrm{E}$, conforme a DRI. Todas as pacientes tiveram consumo de Vitamina C superior ao recomendado.

A Tabela 2 mostra, de forma ordenada, os alimentos que se apresentam como maiores fontes de vitaminas $\mathrm{A}$, $\mathrm{C}$ e $\mathrm{E}$, entre os analisados, e o número de indivíduos que relataram seu consumo. Sobre os alimentos mais ricos em vitamina A, destacaram-se a batata-doce e a cenoura cozida, citadas por mais de $80 \%$ dos pacientes. Em relação à vitamina $\mathrm{C}$, os alimentos mais citados foram batata-doce e couve. Enquanto os mais citados ricos em vitamina $\mathrm{E}$ foram o óleo de soja e a margarina com sal.

Tabela 1. Consumo de vitaminas antioxidantes por pacientes com câncer de mama submetidas à quimioterapia. Pelotas, RS (N=23)

\begin{tabular}{c|c|c}
\hline $\begin{array}{c}\text { Vitaminas } \\
\text { Antioxidantes }\end{array}$ & $\begin{array}{c}\text { Valores de ingestão } \\
\text { Mediana (p25-p75) }\end{array}$ & DRI \\
\hline Vit A $(\mu \mathrm{g} / \mathrm{d})$ & $\begin{array}{c}934,69 \\
(409,24-1542,22)\end{array}$ & 700 \\
\hline Vit C (mg/d) & $\begin{array}{c}250,36 \\
(131,02-498,72)\end{array}$ & 75 \\
\hline Vit E (mg/d) & $\begin{array}{c}3,16 \\
(2,32-5,42)\end{array}$ & 15 \\
\hline
\end{tabular}

DRI $=$ Dietary Reference Intakes

Fonte: Padovani et al. (2006).

Tabela 2. Frequência do consumo de alimentos fontes de vitaminas antioxidantes. Pelotas, $\mathrm{RS}(\mathrm{N}=23)$

\begin{tabular}{c|c|c|c}
\hline Vitamina & Alimento & $\mathbf{N}$ & $\%$ \\
\hline \multirow{4}{*}{$A$} & Carnes & 11 & 47,8 \\
\cline { 2 - 4 } & Manteiga & 4 & 17,4 \\
\cline { 2 - 4 } & Cenoura cozida & 19 & 82,6 \\
\cline { 2 - 4 } & Batata-doce cozida & 21 & 91,3 \\
\hline \multirow{4}{*}{ C } & Couve crua & 17 & 73,9 \\
\cline { 2 - 4 } & Suco de laranja & 16 & 69,6 \\
\cline { 2 - 4 } & Mamão & 11 & 47,8 \\
\cline { 2 - 4 } & Batata-doce cozida & 21 & 91,3 \\
\hline \multirow{4}{*}{ E } & Margarina light & 3 & 13,0 \\
\cline { 2 - 4 } & Azeite de oliva & 1 & 4,3 \\
\cline { 2 - 4 } & Óleo de soja & 22 & 95,7 \\
\cline { 2 - 4 } & Margarina com sal & 16 & 69,6 \\
\hline
\end{tabular}

\section{DISCUSSÃO}

O dano oxidativo pode ocasionar modificaçóes estruturais do DNA, resultando em modificaçōes em suas bases e, consequentemente, na função celular, podendo levar à iniciação e progressão da carcinogênese ${ }^{12-14}$. Dessa forma, tanto a falta de capacidade de reparo ao DNA, diminuição das defesas antioxidantes enzimáticas e não enzimáticas ou o próprio dano causado pelas ERO podem estar envolvidos com o desenvolvimento do câncer, sendo esse efeito potencialmente prevenido por meio do consumo de antioxidantes ${ }^{14,15}$. Por esse contexto, especificamente as vitaminas A, C e E têm sido estudadas em função do seu poder antioxidante ${ }^{13} \mathrm{em}$ pacientes oncológicos $^{16}$.

Um estudo recente de caso controle conduzido nos Estados Unidos, que investigou 5.707 pacientes com câncer de mama, comprovou que um alto consumo de carotenoides pode reduzir significativamente o risco de câncer de mama, principalmente em mulheres pós-menopausa ${ }^{17}$. Sabe-se também que o mecanismo protetor da vitamina $A$ na carcinogênese pode estar relacionado à regulação da diferenciação celular, prevenindo o aumento quantitativo das células com características malignas ${ }^{18}$. Esse conhecimento reforça a importância de um consumo adequado da vitamina $\mathrm{A}$ náo somente para auxiliar na prevenção do câncer de mama, mas também para impedir sua progressão, auxiliando, portanto, no tratamento.

No presente estudo, a mediana de consumo da vitamina A ficou acima do recomendado, sendo que a minoria das pacientes analisadas apresentou baixo consumo. Entretanto, esses resultados devem ser interpretados com cautela. Ainda que a maior parte das mulheres tenha atingido o consumo adequado de vitamina A, um número expressivo de mulheres (mais de 40\%) apresentou baixa ingestão do antioxidante, indicando que as mulheres em quimioterapia devem ser orientadas a ingerir alimentos fonte de vitamina $\mathrm{A}$, a fim de adequar o seu consumo.

A carne, principal fonte dietética investigada de vitamina $\mathrm{A}$, foi referenciada por menos da metade das pacientes, possivelmente por ser um alimento com maior custo quando comparado às outras fontes alimentares. Por outro lado, a cenoura crua e a batata-doce, também fontes alimentares importantes de vitamina $\mathrm{A}$, foram referenciadas pela maioria das pacientes. Isso indica que, para as pacientes com câncer de mama atendidas pelo SUS, é necessário atenção especial, voltada para alimentos populares, fontes da vitamina, que possam ser facilmente inseridos na rotina alimentar.

O consumo de vitamina A varia muito conforme a região, em função da cultura e da acessibilidade a alimentos fontes; por exemplo, um estudo realizado na Coréia $^{19}$, em 2003, observou que a maioria das pacientes consumiu entre $1.553,24$ e $2.702,39 \mu \mathrm{g} / \mathrm{d}$ da vitamina, um consumo muito acima do observado neste estudo. Um estudo caso-controle, na Malásia ${ }^{20}$, demonstrou que além do consumo de vitamina A ser bastante inferior nas pacientes com câncer de mama, quando comparado 
aos controles, essas pacientes também apresentam mais marcadores séricos de agentes oxidativos. Outro estudo realizado em Belo Horizonte (MG), Brasil, com 31 pacientes com câncer de mama, averigou que a maior parte das pacientes apresentava consumo de vitamina A inferior à recomendação ${ }^{21}$. Recomenda-se, portanto, que para a efetiva comparaçáo desses resultados e posterior implantação de condutas nutricionais padronizadas, novos estudos sejam realizados em outros centros de referência.

Em relaçáo ao consumo de vitamina $\mathrm{C}$, um estudo de coorte realizado na Suécia, com 3.405 pacientes com câncer de mama, demonstrou que o consumo da vitamina está associado à maior sobrevida dessas pacientes. No estudo brasileiro de Oliveira et al. (2014) $)^{21}$, a maioria das pacientes demonstrou consumo adequado de vitamina $\mathrm{C}$ (67\%), também avaliado por meio de um Questionário de Frequência Alimentar. No presente estudo, todas as pacientes apresentaram consumo adequado dessa vitamina. As principais fontes dietéticas referidas foram couve crua e suco de laranja, alimentos comuns e de fácil acesso pela maioria da população dessa regiáo.

Uma revisão recentemente publicada concluiu que a vitamina $\mathrm{E}$ pode contribuir para inibição da tumorigênese, uma vez que tem capacidade antioxidante, anti-inflamatória, pró-apoptótica ${ }^{22}$ e alta afinidade por receptor de estrogênio ${ }^{23}$. Assim como o consumo de vitamina $A$, o consumo da vitamina $E$ parece divergir considerando diferentes localidades. Em um estudo na Malásia, por exemplo, o consumo da vitamina atingiu a média de $6,1 \pm 2,4 \mathrm{mg} / \mathrm{d}^{18}$. Já um estudo na Coréia, em 2003, mostrou que a maior parte dos pacientes consumiu entre 6,26 e $12,71 \mathrm{mg} / \mathrm{d}$ de vitamina $\mathrm{E}^{19}$. Ambos os estudos mostram resultados acima daquele observado nas pacientes de Pelotas submetidas à quimioterapia. As fontes de vitamina $\mathrm{E}$ mais referidas pelas pacientes desse estudo foram óleo de soja e margarina com sal, embora as principais fontes investigadas, ou seja, as fontes que continham maior teor dessa vitamina fossem a margarina light e o azeite de oliva. Cabe ressaltar ainda que a baixa ingestáo dietética da vitamina, observada pelo QFA não reflete, necessariamente, na deficiência sanguínea da mesma. Por isso, novos estudos que avaliem a biodisponibilidade desse nutriente podem auxiliar na formulaçáo de recomendaçóes padronizadas para adequação do consumo de vitamina E nessas pacientes.

Em vista da baixa ingestão de vitaminas A e E neste estudo, sugere-se realizar o aconselhamento nutricional para adequar o consumo de alimentos fontes dessas vitaminas e uma investigação dos seus níveis séricos para avaliar a necessidade de suplementaçáo. Um estudo realizado com 2.264 mulheres com diagnóstico de câncer de mama, pertencentes à coorte Life After Cancer Epidemiology, entre 1997 e 2000, que investigou os efeitos da suplementação de antioxidantes pelas pacientes, mostrou associação entre a suplementação de vitaminas C e E com a diminuição do risco de recidiva local, enquanto a suplementaçáo com carotenoides aumentou a mortalidade ${ }^{24}$. Outro estudo de coorte, realizado na China em 2011, concluiu que a suplementação vitamínica pode diminuir o risco de mortalidade e recidiva da doença ${ }^{25}$. Como ainda permanece inconclusiva a eficácia da suplementação vitamínica nessa população, mais investigações para indicação de doses adequadas de consumo e suplementação de antioxidantes que exerçam efeitos benéficos às pacientes com câncer de mama, bem como a identificação do período e duração ideal para prescrição de suplementaçáo, devem ser realizadas. No entanto, a recomendaçáo do aumento do consumo de alimentos ricos em vitaminas antioxidantes é recomendada e deve ser realizada como parte das orientações nutricionais de rotina.

Como limitaçóes do estudo, aponta-se o pequeno tamanho amostral; a falta de informação sobre a composição centesimal dos alimentos avaliados em uma única tabela; a necessidade de utilização de rótulos de certos alimentos; a falta de um QFA específico para avaliação de consumo de antioxidantes dietéticos validado; a análise apenas de vitaminas antioxidantes, sem levar em consideração outros compostos antioxidantes, como o selênio, zinco e polifenóis, a sazonalidade dos alimentos e a utilização de tabelas internacionais para alimentos tipicamente brasileiros. Em relação à avaliação do consumo alimentar, o uso do QFA depende da memória dos entrevistados e pode levar à subestimação do real consumo alimentar. Cabe ressaltar ainda a ausência de um grupo controle para possíveis comparaçóes do consumo de antioxidantes entre mulheres saudáveis e aquelas com câncer de mama.

Apesar das limitaçóes, este foi o primeiro estudo da regiấo que avaliou o consumo de vitaminas antioxidantes em mulheres atendidas pelo SUS submetidas ao tratamento quimioterápico. Estudos sobre o consumo de antioxidantes dietéticos, não só de vitaminas, ainda são escassos na literatura, evidenciando a necessidade de mais pesquisas. Sugere-se que mais estudos investiguem a possibilidade de indicação de fontes dietéticas de vitaminas antioxidantes em alimentos mais populares, bem como a elaboração, padronização e validação de questionários específicos para quantificação de consumo de alimentos antioxidantes para pesquisas científicas futuras.

\section{CONCLUSÃO}

A maior parte da amostra apresentou consumo adequado de vitamina $\mathrm{A}$, embora um número expressivo de pacientes tenha apresentado baixo consumo do nutriente. Todas as pacientes avaliadas apresentaram consumo acima da recomendação para vitamina $\mathrm{C}$ e 
todas consumiram menos do que a recomendação ideal de vitamina E. A adequação dietética desses nutrientes, importantes antioxidantes da dieta, pode contribuir para neutralizar o perfil pró-oxidativo da doença e do tratamento. Evidencia-se, dessa forma, a necessidade de orientaçóes específicas para consumo de alimentos fontes desses antioxidantes com intuito de auxiliar no tratamento e melhorar o estado nutricional dessas pacientes.

\section{CONTRIBUIÇÕES}

Todos os autores participaram efetivamente na concepção, análise e interpretação dos dados e na redação final do manuscrito.

Declaraçáo de Conflito de Interesses: Nada a Declarar.

\section{REFERÊNCIAS}

1. Instituto Nacional de Câncer José Alencar Gomes da Silva. Estimativa 2014: incidência de câncer no Brasil. Rio de Janeiro: Inca; 2014.

2. Ministério da Saúde (BR). Manual de Bases Técnicas de Oncologia - SIA/SUS - Sistema de Informações Ambulatoriais. Brasília, DF: Ministério da Saúde; 2011.

3. Instituto Nacional de Câncer (BR). Ações de enfermagem para o controle do câncer: uma proposta de integração ensino-serviço. Rio de Janeiro: Inca; 2008.

4. Halliwell B. Biochemistry of oxidative stress. Biochem Soc Trans. 2007 Nov;35(Pt 5):1147-50.

5. Suhail N, Bilal N, Khan HY, Hasan S, Sharma S, Khan $\mathrm{F}$, et al. Effect of vitamins $\mathrm{C}$ and $\mathrm{E}$ on antioxidant status of breast-cancer patients undergoing chemother Effect of vitamins $\mathrm{C}$ and $\mathrm{E}$ on antioxidant status of breast-cancer patients undergoing chemotherapy. J Clin Pharm Ther. 2012 Feb;37(1):22-6.

6. Rockenbach G, Di Pietro PF, Ambrosi C, Boaventura BC, Vieira FG, Crippa CG, et al. Dietary intake and oxidative stress in breast cancer: before and after treatments. Nutr Hosp. 2011 Jul-Aug;26(4):737-44.

7. Ribeiro $\mathrm{AB}$, Cardoso MA. Construção de um questionário de freqüência alimentar como subsídio para programas de prevenção de doenças crônicas não transmissíveis. Rev Nutr. 2002 Maio-Ago;15(2):239-45.

8. Padovani RM, Amaya-Farfán J, Colugnati FAB, Domene SMA. Dietary reference intakes: aplicabilidade das tabelas em estudos nutricionais. Rev Nutr. 2006 Nov-Dec; 19(6):741-60.

9. Universidade Estadual de Campinas, Núcleo de Estudos e Pesquisas em Alimentação. Tabela brasileira de composição de alimentos [Internet]. $4^{a}$ edição revisada e ampliada. Campinas: Nepa; 2011 [acesso em 2014 Dez 18]. Disponível em: http://www.unicamp.br/nepa/taco/ contar/taco_4_edicao_ampliada_e_revisada.
10. Universidade Federal de São Paulo, Escola Paulista de Medicina, Departamento de Informática. Tabela de composição química dos alimentos [Internet]. São Paulo: Unifesp; [acesso em 2014 Jan 31]. Disponível em: http:// www.unifesp.br/dis/servicos/nutri/.

11. United States Department of Agriculture, Agriculture Research Service National Nutrient Database for Standard Reference Release 27 [Internet]. Washington, D.C.: USDA; [acesso em 2014 Jan 31]. Disponível em: http://ndb.nal.usda.gov/ndb/search/list.

12. Olinski R, Gackowski D, Foksinski M, Rozalski R, Roszkowski K, Jaruga P. Oxidative DNA damage: assessment of the role in carcinogenesis, atherosclerosis, and acquired immunodeficiency syndrome. Free Radic Biol Med. 2002 Jul 15;33(2):192-200.

13. Yeon JY, Suh YJ, Kim SW, Baik HW, Sung CJ, Kim HS, et al. Evaluation of dietary factors in relation to the biomarkers of oxidative stress and inflammation in breast cancer risk. Nutrition. 2011 Sep;27(9):912-8.

14. Abranches MV, Mendes MCS, Pena GD, Maia YCP, Ribeiro SMR, Franceschini SCC, et al. Antioxidant vitamins and cytokines are altered in breast cancer. Eur J Cancer Prev. 2011 Sep;20(5):403-10.

15. Valtueña S, Pellegrini N, Franzini L, Bianchi MA, Ardigò D, Del Rio D, et al. Food selection based on total antioxidant capacity can modify antioxidant intake, systemic inflammation, and liver function without altering markers of oxidative stress. Am J Clin Nutr. 2008 May;87(5):1290-7.

16. Shah FD, Patel JB, Shukla SN, Shah PM, Patel PS. Evaluation of plasma non-enzymatic antioxidants in breast cancer etiology. Asian Pac J Cancer Prev. 2009 Jan-Mar;10(1):91-6.

17. Mignone LI, Giovannucci E, Newcomb PA, TitusErnstoff L, Trentham-Dietz A, Hampton JM, et al. Dietary carotenoids and the risk of invasive breast cancer. Int J Cancer. 2009 Jun 15;124(12):2929-37.

18. Kim KN, Pie JE, Park JH, Park YH, Kim HW, Kim MK. Retinoic acid and ascorbic acid act synergistically in inhibiting human breast cancer cell proliferation. J Nutr Biochem. 2006 Jul;17(7):454-62.

19. Do MH, Lee SS, Jung PJ, Lee MH. Intake of dietary fat and vitamin in relation to breast cancer risk in Korean women: a case-control study. J Korean Med Sci. 2003 Aug; 18(4):534-40.

20. Sharhar S, Normah H, Fatimah A, Fadilah RN, Rohi GA, Amin I, et al. Antioxidant intake and status, and oxidative stress in relation to breast cancer risk: a case-control study. Asian Pac J Cancer Prev. 2008 Apr-Jun;9(2):343-49.

21. Oliveira DR, Carvalho ESC, Campos LC, Leal JA, Sampaio EV, Cassali GD. Avaliação nutricional de pacientes com câncer de mama atendidas no Serviço de Mastologia do Hospital das Clínicas, Belo Horizonte (MG), Brasil. Ciênc saúde coletiva; 2014 May;19(5):1573-80. 
22. Smolarek AK, Suh N. Chemopreventive activity of vitamin $E$ in breast cancer: a focus on $\gamma$ - and $\delta$-tocopherol. Nutrients. 2011 Nov;3(11):962-86.

23. Nesaretnam K1 Meganathan P, Veerasenan SD, Selvaduray KR. Tocotrienols and breast cancer: the evidence to date. Genes Nutr. 2012 Jan;7(1):3-9.

24. Greenlee H, Kwan ML, Kushi LH, Song J, Castillo A, Weltzien E, et al. Antioxidant supplement use after breast cancer diagnosis and mortality in the Life After Cancer Epidemiology (LACE) cohort. Cancer. 2012 Apr 15;118(8):2048-58.

25. Nechuta S, Lu W, Chen Z, Zheng Y, Gu K, Cai $\mathrm{H}$, et al. Vitamin supplement use during breast cancer treatment and survival: a prospective cohort study. Cancer Epidemiol Biomarkers Prev. 2011 Feb;20(2):262-71. 


\section{Abstract}

Introduction: Breast cancer is the most common type of cancer in women, and the South region of Brazil shows one of the highest rates in the country: it affects more than 70 per 100,000 women. Objective: To evaluate the intake of antioxidant vitamins by women with breast cancer undergoing chemotherapy and to verify the need to adequate their consumption. Method: Cross-sectional study including patients with breast cancer undergoing chemotherapy in the Oncology Department of the Teaching Hospital of Federal University of Pelotas, assisted during the period from May to December of 2012. The dietary intake of patients was assessed by a Food Frequency Questionnaire. Results: The sample consisted of 23 women, $78.3 \%$ adults and $21.7 \%$ elderly. The average age was $56.91 \pm 12.25$ years old. About $43 \%$ of the sample did not reach the recommended intake rate of Vitamin A, although the average was higher than recommended. Concerning Vitamin C, all women reached the recommended intake rate, while no one consumed the minimum amount of vitamin E. Conclusion: It was observed that there is a decreased intake of vitamin $\mathrm{E}$ by all patients, and a significant number of women with low intake of vitamin A. Those are important dietary antioxidants that can help neutralizing the pro-oxidative disease profile. There's a perceived need for nutrition counseling in order to adjust the consumption of antioxidant vitamins in their diet, aiming to assist those patients in the treatment and to improve their nutritional status.

Key words: Breast Neoplasms; Antioxidants; Drug Therapy; Humans; Female; Brazil

\section{Resumen}

Introducción: El cáncer de mama es el más común entre las mujeres, y el Sur tiene uno de los índices más altos del país: afecta a más de 70 por cada 100.000 mujeres. Objetivo: Evaluar el consumo de vitaminas antioxidantes por parte de mujeres con cáncer de mama en tratamiento quimioterápico y verificar la necesidad de adecuar su consumo. Método: Estudio transversal con pacientes con neoplasia mamaria en quimioterapia en el Sector de Oncología del Hospital Escola da Universidade Federal de Pelotas (Hospital Escuela de la Universidad Federal de Pelotas), atendidas entre mayo y diciembre del 2012. El consumo alimentar de las pacientes fue evaluado por medio de un Cuestionario de Frecuencia Alimentar. Resultados: La muestra está formada por 23 mujeres, $78.3 \%$ adultos y $21.7 \%$ de ancianos. El promedio de edad fue de 56,91 $\pm 12,25$ ańos. Aproximadamente el 43\% de la muestra no alcanzó el valor recomendado de ingestión de Vitamina A, aunque el promedio de consumo haya sido mayor que lo recomendado. En relación a la Vitamina C, todas ultrapasaron lo ideal, por otro lado, consumieron la cantidad mínima de vitamina E. Conclusión: Se observó que hay una disminución de la ingesta de vitamina $\mathrm{E}$ por parte de todas las pacientes, y un número significativo de mujeres con baja ingesta de vitamina A, importantes antioxidantes en la dieta que pueden ayudar a neutralizar el perfil pro oxidativo de la enfermedad. Se detectó la necesidad de orientación nutricional con el objetivo de adecuar el consumo de vitaminas antioxidantes en la dieta para auxiliar al tratamiento y mejorar el estado nutricional de esas pacientes. Palabras clave: Neoplasias de la Mama; Antioxidantes; Quimioterapia; Humanos; Feminino; Brasil 